American Journal of Pediatrics
2020; 6(4): $448-454$
http://www.sciencepublishinggroup.com/j/ajp
doi: 10.11648 /j.ajp.20200604.20
ISSN: $2472-0887$ (Print); ISSN: $2472-0909$ (Online)

\title{
A Clinical Profile and Immediate Outcome of Acute Glomerulonephritis (AGN) in Hospitalized Children
}

\author{
A. N. M. Shahidul Islam Bhuiyan ${ }^{1, ~ *}$, Zahangir Alam², Khondaker Zahirul Hasan², \\ Md Mostafa Zaman ${ }^{3}$, Md Zakaria ${ }^{4}$ \\ ${ }^{1}$ Department of Paediatrics, Cumilla Medical College, Cumilla, Bangladesh \\ ${ }^{2}$ Department of Paediatrics, Abdul Malek Ukil Medical College, Noakhali, Bangladesh \\ ${ }^{3}$ Department of Paediatrics, M Abdur Rahim Medical College, Dinajpur (MARMC), Bangladesh \\ ${ }^{4}$ Department of Paediatrics, Sunamgong Sadar Hospital, Sunamgong, Sylhet, Bangladesh
}

\section{Email address:}

riponbhuiyan.bd@gmail.com (A. N. M. S. I. Bhuiyan)

*Corresponding author

\section{To cite this article:}

A. N. M. Shahidul Islam Bhuiyan, Zahangir Alam, Khondaker Zahirul Hasan, Md Mostafa Zaman, Md Zakaria. A Clinical Profile and Immediate Outcome of Acute glomerulonephritis (AGN) in Hospitalized Children. American Journal of Pediatrics.

Vol. 6, No. 4, 2020, pp. 448-454. doi: 10.11648/j.ajp.20200604.20

Received: July 26, 2020; Accepted: October 13, 2020; Published: November 4, 2020

\begin{abstract}
Introduction: Acute glomerulonephritis includes renal diseases in which immunologic mechanism triggers inflammation and proliferation of glomerular tissue that can result in damage to the basement membrane, proliferation of mesangium, capillary endothelium. Objectives: The objective of this study is to know the clinical profile and immediate outcome of acute glomerulonephritis in hospitalized children. Material \& Methods: The study was done in Chittagong Medical College Hospital Pediatric Unit during the period of June 2007 to February 2008. All patients admitted with AGN, with or without complications were included in this study. Diagnostic criteria were scanty urine (infrequent and less than normal in amount as stated by the parents), swelling, high colored urine with or without albuminuria, no past history of similar attack and microscopic or naked eye haematuria. Criteria of discharge from the hospital were absence of puffiness and oedema, adequate urine formation, absence of heart failure and hypertensive encephalopathy. These were taken as clinical recovery. No long-term follow up was done. Results: Seventy-eight cases of acute glomerulonephritis (AGN) in children under 12 years of age were studied. Male to female ration 3:2. Scanty urine $(84.0 \%)$, puffy face $(88.5 \%) \mathrm{h}$, haematuria $(80.0 \%)$, hypertension $(82.5 \%)$, heart failure (11.5\%) convulsion (14\%) anuria (3.8\%), RBC (92.3\%), RBC cast $(41 \%)$, albumin one $(+)(52.6 \%)$ two $+(14.1 \%)$ three $+(14.1 \%)$, raised s. creatinine was $(25.6 \%)$, blood urea $(26.9 \%)$. Four patients were died. Among them three was due to hypertension and heart failure. One due to the development of acute renal failure. History of skin infection like scabies was present in $61.4 \%$ patient. Conclusion: Skin infection is the commonest cause of acute glomerulonephritis. Nephritic presentation (scanty urine oedema, haematuria, hypertension and heart failure) was the commonest mode of presentation. Immediate prognosis was excellent- Long term follow up is recommended.
\end{abstract}

Keywords: Acute Glomerulonephritis, Skin Infection, Hospitalized Children, Pediatric, Haematuria

\section{Introduction}

Acute glomerulonephritis includes renal diseases in which immunologic mechanism triggers inflammation and proliferation of glomerular tissue that can result in damage to the basement membrane, proliferation of mesangium, capillary endothelium. Hippocrates originally described the manifestation of back pain and hematuria, which lead to oliguria or anuria. With the development of the microscope, Langhans was later able to describe these pathophysiologic glomerular disorder affecting children and also is an important cause of hospital admission in peadiatric unit. Acute glomerulonephritis, particularly acute poststreptococcal glomerulonephritis, in children is a common condition caused due to bacterial infection with group-A beta hemolytic streptococcus typically involving a 
throat, upper respiratory tract and skin [1]. Acute post streptococcal glomerulonephritis affects children between the ages of five and twelve years and usually rare before the age of three [2]. Most research focuses on the post-streptococcal origin. Acute glomerulonephritis is defined as the sudden onset of hematuria, proteinuria, and red blood cell casts. The clinical picture is often accompanied by hypertension, edema, and impaired renal function. glomerulonephritis refers to groups of renal disease characterized by inflammation of glomerulus manifested by proliferation of cellular elements secondary to immunogenic mechanism. Secondary forms of glomerulonephritis are much more common in the topics than in the industrialized countries [3]. It is more prevalent among the population particularly where poverty overcrowding, poor hygiene living is prevailing, this particular disease contributes to a significant amount of disease burden particularly on hospital admission and the major primary cause is infection [4]. Some of the other less common infective agents have also been identified, such as Streptococcus, schistosomiasis and malaria. Others are only speculative, being suspected from the strikingly high prevalence of proliferative glomerulonephritis, the prototype lesion of such etiology. The clinical picture is quite obvious but laboratory evidences of recent streptococcal infection by finding of elevated anti-streptococcal antibodies and reduction in serum complement levels due to its consumption in antigen-antibody interaction in the kidneys lend additional diagnostic support. Regarding management and prognosis, the immediate outcome is rather good in children, but the long-term prognosis is poor. Mortality in acute stage varies and they result from circulatory congestion due to heart failure hypertensive encephalopathy and acute renal failure [6]. The glomerular infection and extension damages the microcirculation, by dipping the glomerular filtration rate (GFR) and generally causes an increase in BUN and creatinine. This decrease in GFR, sequentially leads to the preservation of salt and water and produce fluid overload. The amount of fluid overload in AGN might be differ from substantially. In severe circumstances, it might be visible by serious hypertension and pulmonary edema. Impulsive determination of the clinical indicators is mostly quick: diuresis commonly results in within 1-2 weeks, and the serum creatinine concentration returns to base line within 4 weeks. The degree at which point urinary abnormalities dissolve is not less inconstant. Hematuria generally resolves by 6 months, but insignificant proteinuria is existent in $15 \%$ of patients after 3 years and in $2 \%$ of patients after 10 years. [6]

\section{Objectives}

General objective

To assess the clinical profile and immediate outcome of acute glomerulonephritis in hospitalized children.

Specific Objectives

1. To know presenting features of acute GN.

2. To assess the course of disease among hospitalized acute glomerulonephritis patients

3. To ascertain the immediate outcome among hospitalized acute glomerulonephritis patients.

4. To assess the socio demographic pattern of these children.

\section{Methodology and Materials}

The study was done in Chittagong Medical College Hospital Pediatric Unit during the period of June 2007 to February 2008. All patients admitted with AGN, with or without complications were included in this study. Recruited sample size was 83 , five of the patients were excluded from the analysis due to incompleteness of the data hence 78 cases were selected for analysis. Diagnostic criteria were scanty urine (infrequent and less than normal in amount as stated by the parents), swelling, high colored urine with or without albuminuria, no past history of similar attack and microscopic or naked eye haematuria. Criteria of discharge from the hospital were absence of puffiness and oedema, adequate urine formation, absence of heart failure and hypertensive encephalopathy. These were taken as clinical recovery. No long-term follow up was done.

Inclusion Criteria

1. Children admitted with Acute glomerulonephritis in to paediatric unit of $\mathrm{CMCH}$

2. Stayed in the hospital and received at least 7 days treatment

3. Willing to participate in the study

Exclusion Criteria

1. Patients with other systemic disease unrelated to acute glomerulonephritis

2. Not willing to participate

\section{Results}

Table 1 shows the distribution of the respondents by age. Among the acute glomerulonephritis patients 9.0\% aged less than 3 years, $20.5 \%$ aged between $4-6$ years, and $39.7 \%$ aged between $7-9$ years and another $30.8 \%$ were aged between $10-12$ years. Figure 1 shows the distribution of the study patients by sex. Among the children with acute glomerulonephritis $60 \%$ were male and $40 \%$ were female. Figure 2 show the distribution of the study patients by place of residence. Among the acute glomerulonephritis patient $58 \%$ were from urban place of residence, $23 \%$ were from semi urban back ground and 19\% were from rural areas. Figure 3 illustrates the distribution of the study patients by their socioeconomic status. Most of the $(59 \%)$ patients belong to the Low socioeconomic status, $17.9 \%$ were with middle socioeconomic status and only $23.1 \%$ share high socioeconomic status. Among the acute glomerulonephritis patients in the study $42.1 \%$ provided the history of Sore throat, $61.4 \%$ provided the history of infected scabies and $19.3 \%$ reported previous exposure to both Sore throat \& skin infection. 'Multiple response analysis' was performed to find out the prevalence of individual presenting feature among the acute glomerulonephritis patients. Among the most striking 
features puffy face $(88.5 \%)$, Hematuria $(80.7 \%)$ and Scanty micturition $(84.0 \%)$ were most prevalent in different combination. Of the total patients $(23.1 \%)$ had respiratory distress, $24.4 \%$ had cough, and other complications wereheadache $(17.9 \%)$, vomiting $(14.1 \%)$, chest pain $(14.1 \%)$, convulsion (14.1\%). Among the other generalized features fever $(7.7 \%)$, abdominal pain $(2.6 \%)$ were also reported. Presenting general examination finding of the acute glomerulonephritis patient reported in paediatric hospital were tabulated in Table 4. Of the total participants pallor was found in $57.0 \%$ patients, oedema was found in $63.5 \%$ children with acute glomerulonephritis and hypertension was found in $82.5 \%$ patients. $11.5 \%$ had enlarged liver, $14.1 \%$ Lung crepitations, and $6.3 \%$ had engorged neck vein. Table 5 shows the urinary findings of acute glomerulonephritis patients. Regarding, urinary manifestations among the study patients in 24 hours $3.8 \%$ had anuria. Urinary volume was $<250 \mathrm{ml}$ in $10.3 \%$ patients. $250-500 \mathrm{ml} 9.0 \%$, 500-750ml$39.7 \mathrm{R}$ and above $750 \mathrm{ml}$ in $37.2 \%$ cases. Urinary protein was measured and in $19.2 \%$ patient no protein was detected. In $52.6 \%$ patient protein content was One + in $14.1 \%$ protein content was 'two +' and in $14.1 \%$ patient protein content was 'three + '. Following routine microscope examination RBC was detected in $92.3 \%$ patients, Pus cell was detected in $20.5 \%$ patient, RBC cast was detected in $41.0 \%$ patient, granular cast was found in $17.9 \%$ patients and Hyaline cast was detected $5.1 \%$ patient. Culture of urine revealed no growth in $91.03 \%$ cases and growth of E coli was found in $8.97 \%$ cases. Erythrocyte sedimentation rate (ESR) examination revealed $>20 \mathrm{~mm}$ in $1^{\text {st }}$ hour in $87.2 \%$ of the subjects. Raised ASO titre ( $>200 \mathrm{IU})$ was found in $52.6 \%$ patients, raised urea in blood $(>60 \mathrm{mg} / \mathrm{dl})$ was found in $26.9 \%$ patients, raised serum creatinine $(>1 \mathrm{mg} / \mathrm{dl})$ was found in $25.6 \%$ patients. Throat swab culture in $80.8 \%$ cases no growth was found, in $5.1 \%$ cases Streptococcus $\beta$ hemolyticus growth was found and in $14.1 \%$ growth of normal flora was found. Following Skin culture in $91.0 \%$ cases no growth was found, in $9 \%$ cases Streptococcus $\beta$ hemolyticus was isolated. Figure 4 shows the distribution of the time required to subside the oedema in patients with acute glomerulonephritis. Among the 54 patients, in $17.9 \%$ case oedema was subsided within 5 days, in $7.7 \%$ cases oedema was subsided between 6 - 10 days, in $15.4 \%$ cases oedema was subsided between $11-15$ days in $2.6 \%$ cases oedema was subsided between 16-20 days and in $2.6 \%$ cases oedema was still persistent at discharge. Figure 5 shows the distribution of the time required to control the hypertension in patients with acute glomerulonephritis. Among the 64 patients with hypertension, in $1.3 \%$ case hypertension was subsided within 5 days, in $20.5 \%$ cases hypertension was subsided between 6 - 10 days, in $12.8 \%$ cases hypertension was subsided between 11 - 15 days in $26.9 \%$ cases hypertension was subsided between 16 - 20 days and in $1.3 \%$ cases hypertension was still persistent at discharge. Figure 6 depicts the distribution of the time required to subside hematuria. Among the patients with Hematuria on admission in $55 \%$ cases haematuria was subsided in between $6-10$ days. In $28 \%$ cases haematuria was subsided in between $11-$ 15 days and in $11 \%$ cases haematuria was subsided in between $16-20$ days. In $6 \%$ cases haematuria was persistent during the discharge. Length of hospital stay for the event of illness or episode of acute glomerulonephritis is illustrated in Table 8. Among the acute glomerulonephritis patient survived the illness episode $14.1 \%$ had to stay in hospital for $<1$ week, $24.4 \%$ had to stay in hospital for $1-2$ weeks, $39.7 \%$ had to stay in hospital for $2-3$ weeks, and $21.8 \%$ had to stay in hospital 3 - 4 weeks. Immediate outcome of the acute glomerulonephritis was tabulated in Table 9, Among the 78 Acute glomerulonephritis patients $57.7 \%$ had compete recovery on discharge, $1.28 \%$ Acute renal failure (ARF), $11.5 \%$ Heart failure, $14.1 \%$ Hypertensive encephalopathy, $10.3 \%$ Discharge own risk bond and 5.1\% Death.

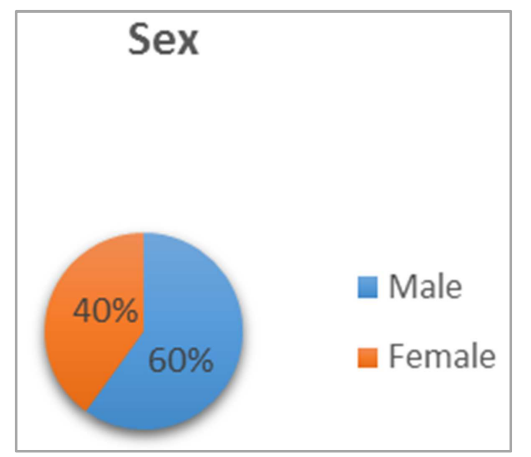

Figure 1. Distribution of the study subjects by sex. (78).

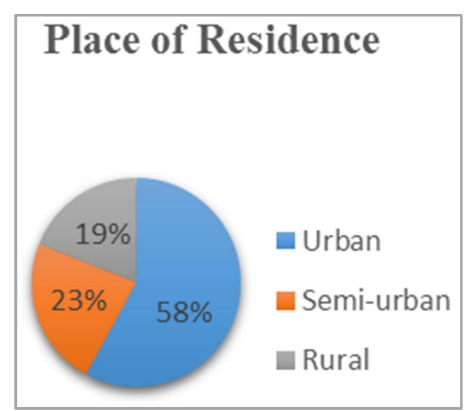

Figure 2. Distribution of the study subjects by place of residence. $(n=78)$.

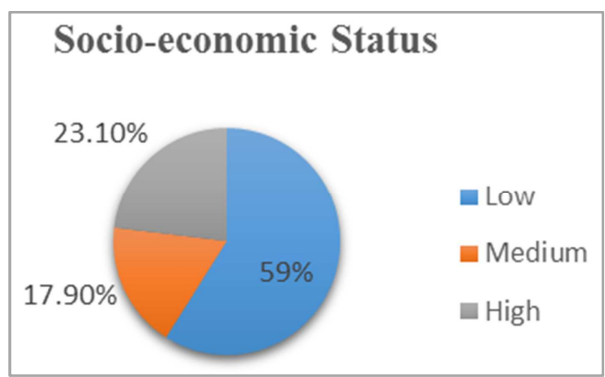

Figure 3. Distribution of the study subjects by Socio economic status. $(n=78)$.

Table 1. Distribution of the study subjects by age. $(n=78)$.

\begin{tabular}{lll}
\hline Age & Frequency & $\mathbf{\%}$ \\
\hline$<3$ Years & 7 & 9.0 \\
$4-6$ Years & 16 & 20.5 \\
$7-9$ Years & 31 & 39.7 \\
$10-12$ Years & 24 & 30.8 \\
Total & 78 & 100.0 \\
\hline
\end{tabular}




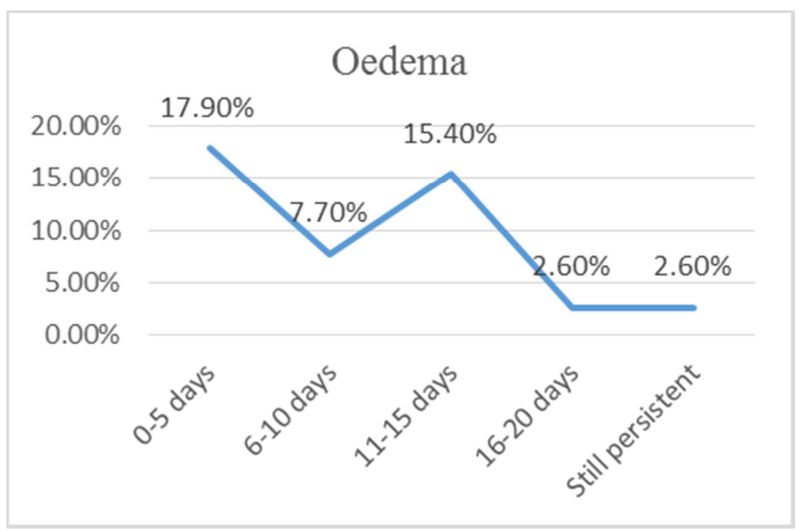

Figure 4. Period of subsiding Oedema. $(n=78)$.

Table 2. Distribution of past history of risk factor exposure. $(n=78)$.

\begin{tabular}{lll}
\hline History of infection & Frequency & $\mathbf{\%}$ \\
\hline Skin infection & 24 & $42.1 \%$ \\
Sore throat & 24 & $42.1 \%$ \\
Infected scabies & 35 & $61.4 \%$ \\
Both Sore throat \& skin infection & 11 & $19.3 \%$ \\
& 94 & $164.9 \%$ \\
\hline
\end{tabular}

* Percentage exceeds 100 dues to multiple responses.

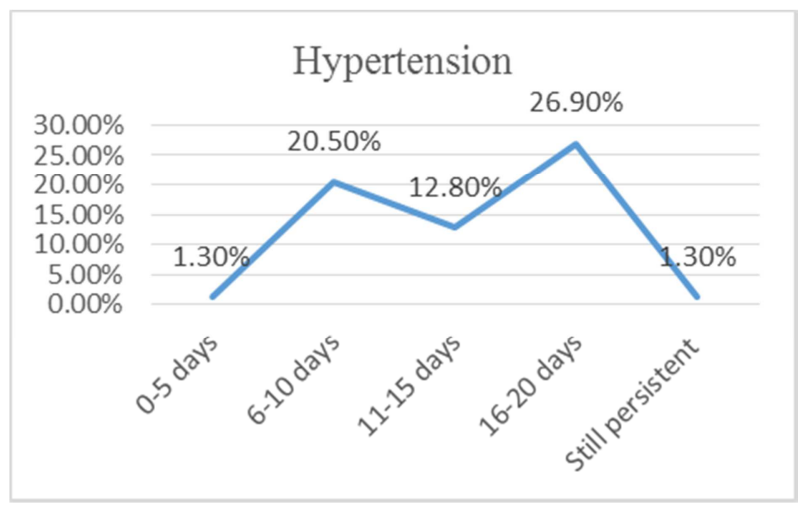

Figure 5. Period of controlling hypertension. $(n=78)$.

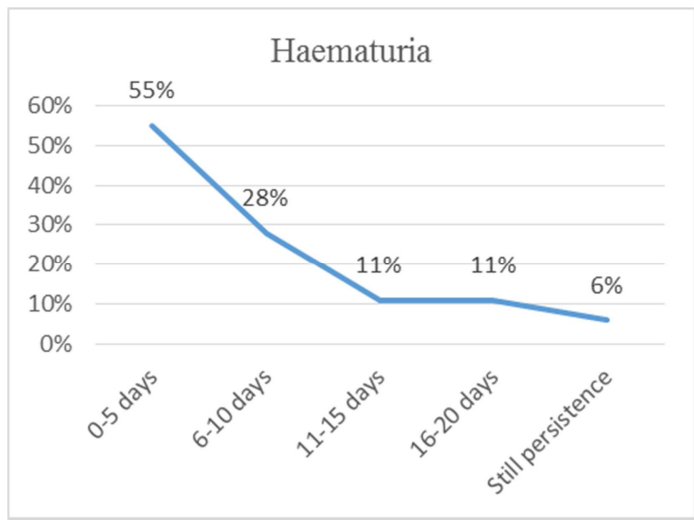

Figure 6. Period of disappearing heamaturia. $(n=78)$.

Table 3. Distribution of presenting features. $(n=78)$.

\begin{tabular}{lll}
\hline Presenting featuresa & Frequency & $\mathbf{\%}$ \\
\hline Puffy face & 69 & 88.50 \\
Scanty micturition & 65 & 84.00 \\
Hematuria & 62 & 80.70 \\
Respiratory Distress & 18 & 23.10 \\
Cough & 19 & 24.40 \\
Headache & 14 & 17.90 \\
Vomiting & 11 & 14.10 \\
Chest Pain & 11 & 14.10 \\
Convulsion & 11 & 14.10 \\
Fever & 6 & 07.70 \\
Abdominal pain & 2 & 02.60 \\
\hline
\end{tabular}

* Percentage exceeds 100 due to multiple responses.

Table 4. Distribution of Clinical features found in general examination. $(n=78)$.

\begin{tabular}{lll}
\hline Clinical feature & Frequency & \% \\
\hline Pallor & 45 & $57.0 \%$ \\
Oedema & 40 & $63.5 \%$ \\
Hypertension & 64 & $82.5 \%$ \\
Enlarged Liver & 09 & $11.5 \%$ \\
Lung crepitations & 11 & $14.1 \%$ \\
Engorged Neck Vein & 04 & $06.3 \%$ \\
\hline
\end{tabular}

* Percentage exceeds 100 due to multiple responses.

Table 5. Distribution of urine analysis findings. $(n=78)$.

\begin{tabular}{lll}
\hline Criteria & Frequency & $\mathbf{\%}$ \\
\hline Urine Volume in $24 \mathrm{hr}$. & & 03.8 \\
Anuria & 03 & 10.3 \\
$<250 \mathrm{ml}$ & 08 & 09.0 \\
$250-500 \mathrm{ml}$ & 07 & 39.7 \\
$500-750 \mathrm{ml}$ & 31 & 37.2 \\
$>750 \mathrm{ml}$ & 29 & \\
Urinary protein & & 19.2 \\
Nil & 15 & 52.6 \\
One + & 41 & 14.1 \\
two + & 11 & 14.1 \\
three + & 11 & \\
Urine RME* & & $92.3 \%$ \\
RBC & 72 & $20.5 \%$ \\
Puss cell & 16 & $41.0 \%$ \\
RBC Cast & 32 & $17.9 \%$ \\
Granular Cast & 14 & $5.1 \%$ \\
Hyaline Cast & 04 & \\
\hline
\end{tabular}




\begin{tabular}{lll}
\hline Criteria & Frequency & \% \\
\hline Urine culture & & \\
No growth & 71 & $91.03 \%$ \\
E. coli & 07 & $8.97 \%$ \\
Total & 78 & 100.0 \\
\hline
\end{tabular}

* Percentage exceeds 100 due to multiple responses.

Table 6. Distribution of finding laboratory investigation. $(n=78)$.

\begin{tabular}{lll}
\hline Lab investigation & Frequency & \% \\
\hline ESR $>20 \mathrm{~mm}$ in $1^{\text {st }}$ hour & 68 & $87.2 \%$ \\
Raised ASO titre & 41 & $52.6 \%$ \\
Raised Urea blood & 21 & $26.9 \%$ \\
Raised Serum creatinine & 20 & $25.6 \%$ \\
\hline
\end{tabular}

(ASO titre $>200 \mathrm{IU} / \mathrm{ml}$, Raised Urea blood $60 \mathrm{mg} / \mathrm{dl}$, Serum creatinine $>1 \mathrm{mg} / \mathrm{dl}$.

Table 7. Distribution of the evidences of active infection. $(n=78)$.

\begin{tabular}{lll}
\hline Specimen & Frequency & \% \\
\hline Throat swab examination & 63 & $80.8 \%$ \\
No growth & 04 & $05.1 \%$ \\
Streptococcus $\beta$-hemolyticus & 11 & $14.1 \%$ \\
Normal flora & & \\
Skin swab examination & 71 & $91.0 \%$ \\
No growth & 07 & $09.0 \%$ \\
Streptococcus $\beta$-hemolyticus & 78 & $100.0 \%$ \\
Total & 78 & \\
\hline
\end{tabular}

Table 8. Distribution of Length of hospital stay. ( $n=78)$.

\begin{tabular}{lll}
\hline Length of hospital stay & Frequency & \% \\
\hline$<1$ Week & 11 & $14.1 \%$ \\
$1-2$ weeks & 19 & $24.4 \%$ \\
$2-3$ weeks & 31 & $39.7 \%$ \\
$3-4$ weeks & 17 & $21.8 \%$ \\
Total & 78 & $100.0 \%$ \\
\hline
\end{tabular}

Table 9. Distribution of immediate outcome of treatment discharge. $(n=78)$.

\begin{tabular}{lll}
\hline Immediate Outcome & Frequency & \% \\
\hline Complete recovery on discharge & 45 & $57.7 \%$ \\
Acute renal failure (ARF) & 1 & $1.28 \%$ \\
Heart failure & 9 & $11.5 \%$ \\
Hypertensive encephalopathy & 11 & $14.1 \%$ \\
Discharge own risk bond & 8 & $10.3 \%$ \\
Death & 4 & $5.1 \%$ \\
Total & 78 & $100.0 \%$ \\
\hline
\end{tabular}

\section{Discussion}

Even though the pathogenesis is not fully understood, existing evidence provisions that most cases of acute glomerulonephritis are due to an immunologic reaction to a variety of different etiologic agents. The immunologic response, sequentially, stimulates a number of biological procedures that cause glomerular inflammation and damage. Acute glomerulonephritis perhaps will be isolated to the kidney or be a factor of a systemic condition. Diseases containing the renal glomeruli are come across habitually in clinical practice and are the most common reasons of end stage renal disease globally [7]. The true incidence of the disease is difficult to ascertain because of the wide range of clinical manifestations. Management of the disease in children is primarily dependent on supportive and symptomatic care and very little can be done to modify the course of the disease. Physicians often face difficulty in managing children with mixed presentation and complication. The present study opted to report the Clinical profile and immediate outcome of children with admission diagnosis of $\mathrm{AGN}$, in Chittagong medical college hospital. The immediate outcome of the disease is favorable in children than in adults. Hence prompt diagnosis in children may ease the difficulty of physicians in treating the disease and could increase the chance of prompt recovery. Moreover, forms of presentation and extent of immediate clinical outcome is worth exploring. For these reasons, it is of considerable interest to identify the patterns of glomerular disease in 
specific regions. Among the acute glomerulonephritis patients $9.0 \%$ aged less than 3 years, $20.5 \%$ aged between $4-6$ years, and $39.7 \%$ aged between $7-9$ years and another $30.8 \%$ were aged between $10-12$ years. Majority $(60 \%)$ of the patients were female. The pattern is identical with other researches. According to Rodriguez-Iturbe ${ }^{2}$ around $58 \%$ were from urban place of residence, $23 \%$ were from semi urban back ground and $19 \%$ were rom rural areas. Regarding socioeconomic status, $17.9 \%$ were with Middle socioeconomic status and only $23.1 \%$ share high socioeconomic status. Other researches also suggest that it is more prevalent among the population where poverty overcrowding, poor hygiene living is prevailing. The major primary cause identified most researchers was infection [8]. Some of the other less common infective agents have also been identified, such as Streptococcus, schistosomiasis and malaria. Others are only speculative, being suspected from the strikingly high prevalence of proliferative glomerulonephritis, the prototype lesion of such etiology History of past streptococcal infection is described as an established risk factor that illustrate causal link of acute glomerulonephritis. In the current study $42.1 \%$ provided the history of skin infection, $42.1 \%$ provided the history of infected scabies and $19.3 \%$ reported previous exposure to both Sore throat \& skin infection. Acute glomerulonephritis presents with some complaints. Following multiple response analysis striking features found scanty urine $(84.0 \%)$ puffy face $(88.5 \%)$ haematuria $(80.7 \%)$ hypertension $(82.5 \%)$, heart failure $(11.5 \%)$, convulsions $(14.1 \%)$, anuria $(3.8 \%)$, headache $(17.9 \%)$, cough $(24.4 \%)$ on physical examination patients among the study participants pallor (57\%), oedema $(69.2 \%)$, hypertension $(82.5 \%)$, enlarged liver (11.5\%). Lung crepitations (14.1\%). Hypertension happens in nearly $80-90 \%$ of cases of acute glomerulonephritis and cerebral complications including headache, seizures, mental status changes and visual changes occur in $30-35 \%$ of cases [9]. In this study, we found hypertension in $82.5 \%$ cases. The commonness of cerebral complications is lower compared to the observations made by other studies, whereas cardiac complications are also comparatively few [10] The high prevalence of hypertension as presenting feature could be due to failure to attend treatment in the earlier stage of illness resulting late sequel of the disease. we found raised ASO titre in $52.6 \%$. Also, serum creatinine level was high only in $25.6 \%$ of the cases. Similar cases have been reported in some studies [11-13].

\section{Conclusion and Recommendations}

Seventy-eight cases of different age groups were admitted during the eight months period, from June 2007 to February 2008. Highest incidence was observed during the months of October, November and December. Incidence of the disease was more among the poor socioeconomic classes. There was male preponderance of the disease in this study. The peak age observed was between 7-9 years. The cases were suspected to be of post-streptococcal origin as evidenced by history of skin infections or sore throat and or raised as titre although B-hemolytic streptococcus could not be isolated in most cases. Occurrence of heart failure was $11.5 \%$. $61.4 \%$ had infected scabies and $82.5 \%$ hypertension. About complete recovery was obtained in $95 \%$ of cases. Hematuria was present in $6 \%$ during discharge. Emphasis should be given on prevention of the disease through health education, providing primary health care and early recognition and treatment of scabies infections and streptococcal pharyngitis. Timely and proper intervention is necessary and long term follow up should be done in every patient.

\section{Limitations of the Study}

The present study was conducted at a very short period of time. Recruited sample size of this study was 83, five of the patients were excluded from the analysis due to incompleteness of the data hence 78 cases were selected for analysis. For being a study in a single community with comparatively small number of sample size, the study result may not reflect the exact scenarios of the whole country.

\section{References}

[1] Glassock RJ, Cohen AH, Adler SG: primary Glomerular disease Brenner Reetor's: The Kidney, $5^{\text {th }}$ edn. Saunders vol$11 \mathrm{p}-1392-1473$.

[2] Rodriguez-Iturbe B, Batsford S. Pathogenesis of poststreptococcal glomerulonephritis a century after Clemens von Pirquet. Kidney Int. 2007; 71 (11): 1094-104.

[3] Batsford SR, Mezzano S, Mihatsch M, et al. Is the nephritogenic antigen in post-streptococcal glomerulonephritis py8rogenic exotoin B (SPE B) or GAPDH? Kidney Int. 2005; 68 (3): 1120-9.

[4] Derakhshan A, Hosseini Al, Hashemi G, Fallahzadeh MH. Spectrum of inpatient renal diseases in children, a report from Southern part of Islamic Republic of Iran. Saudi J Kidney Dis Transplant. 2004; 15 (1): 12.

[5] Chadban SJ, Atkins RC. Glomerulonephritis. Lancet 2005; 365: 1797.

[6] Carapetis JR, Steer AC, Mulholland EK, Weber M. The global burden of group A streptococcal disease. Lancet Infect Dis 2005; 5: 685 .

[7] Rodriguez-Iturbe B. Postinfectious glomerulonephritis. Am J Kidney Dis 2000; 35: XLVI.

[8] Sanjad S, Tolaymat A, Whitworth J, Levin S. Acute glomerulonephritis in children: a review of 153 cases. South Med J 1977; 70: 1202.

[9] Cattran DC. Evidence-Based Recommendations for The Management of Glomerulonephritis. Introduction. Kidney IntSuppl 1999 Jun; 70: S1-2. 12. 
[10] Steer AC, Danchin MH, Carapetis JR. Group A streptococcal infection in children. J Paediatr Child Health 2007; 43: 203 213.

[11] Gumus, H. Per, S. Kumandas, and A. Yikilmaz, "Reversible posterior leukoencephalopathy syndrome in childhood: report of nine cases and review of the literature," Neurological Sciences, vol. 31, no. 2, pp. 125-131, 2010.
[12] T. M. Eison, B. H. Ault, D. P. Jones, R. W. Chesney, and R. J. Wyatt, "Post-streptococcal acute glomerulonephritis in children: clinical features and pathogenesis," Pediatric Nephrology, vol. 26, pp. 165-180, 2011.

[13] R. Bogdanovic, Henoch-Schönlein purpura, "Nephritis in children: risk factors, prevention and treatment," Acta Paediatrica, vol. 98, no. 12, pp. 1882-1889, 2009. 